\title{
Metronidazole-induced encephalopathy in a patient with liver cirrhosis
}

\author{
Hyeong Cheol Cheong, Taek Geun Jeong, Young Bum Cho, Bong Joon Yang, \\ Tae Hyeon Kim, Haak Cheoul Kim, and Eun-Young Cho \\ Department of Internal Medicine, Wonkwang University College of Medicine, Iksan, Korea
}

\begin{abstract}
Encephalopathy is a disorder characterized by altered brain function, which can be attributed to various causes. Encephalopathy associated with metronidazole administration occurs rarely and depends on the cumulative metronidazole dose, and most patients with this condition recover rapidly after discontinuation of therapy. Because metronidazole is metabolized in the liver and can be transported by the cerebrospinal fluid and cross the blood-brain barrier, it may induce encephalopathy even at a low cumulative dose in patients with hepatic dysfunction. We experienced a patient who showed ataxic gait and dysarthric speech after receiving metronidazole for the treatment of hepatic encephalopathy that was not controlled by the administration of lactulose. The patient was diagnosed as metronidazole-induced encephalopathy, and stopping drug administration resulted in a complete recovery from encephalopathy. This case shows that caution should be exercised when administering metronidazole because even a low dose can induce encephalopathy in patients with liver cirrhosis. (Korean J Hepatol 2011;17:157-160)
\end{abstract}

Keywords: Metronidazole; Encephalopathy; Cirrhosis

\section{INTRODUCTION}

Metronidazole is a 5-nitroimidazole derivative and is used clinically to treat Crohn's disease, abdominal abscess, cellulitis, extradural abscess, meningitis, postoperative inflammation, and infection with Helicobacter pylori. ${ }^{1}$ In addition, it is used to treat hepatic encephalopathy that is caused by liver cirrhosis but cannot be controlled by administration of lactulose. ${ }^{2}$ The known adverse effects of the drug include peripheral neuropathy, convulsion, encephalopathy, cerebellar dysfunction, vertigo, ataxia, and dysarthria. ${ }^{3}$ We observed dysarthria and ataxia after administration of metronidazole in a patient with hepatic encephalopathy that could not be controlled by administration of lactulose, and we diagnosed the condition as metronidazole-induced encephalopathy on the basis of brain magnetic resonance imaging (MRI) findings. Drug administration was then stopped, and the metronidazole-induced encephalopathy reversibly improved.
Here, we report a case of metronidazole-induced encephalopathy with a review of the literature.

\section{CASE REPORT}

A 57-year-old man visited the hospital for right chest pain after falling down in the bathroom. He had been hospitalized several times for alcoholic liver cirrhosis with hepatic encephalopathy. He has drunk $160 \mathrm{~g}$ of alcohol everyday for 35 years but had abstained from drinking for the previous 11 months. He had also been taking metronidazole $750 \mathrm{mg} /$ day beginning 10 days before admission because his hepatic encephalopathy was not controlled by administration of lactulose. At admission, his blood pressure was 100/60 $\mathrm{mmHg}$, body temperature was $36.5^{\circ} \mathrm{C}$, pulse rate was $90 / \mathrm{min}$, and respiratory rate was $20 / \mathrm{min}$. In the physical examinations, chronically ill appearance, icteric sclera, traces of bruising and some tenderness on the right thoracic

Received October 7, 2010; Revised March 18, 2011; Accepted April 1, 2011

Abbreviations: MRI, magnetic resonance imaging; AST, aspartate aminotransferase; ALT, alanine aminotransferase; CT, computed tomography; FLAIR, fast fluid-attenuated inversion-recovery

Comesponding author: Eun-Young Cho

Department of Internal Medicine, Wonkwang University College of Medicine, 344-2 Sinyong-dong, Iksan 570-711, Korea

Tel. +82-63-859-2564, Fax.+82-63-855-2025, E-mail; love6970@wonkwang.ac.kr 
wall, splenomegaly of the left upper quadrant, and pitting edema in the peripheral extremities were observed. Blood chemistry tests revealed the following findings: hemoglobin,

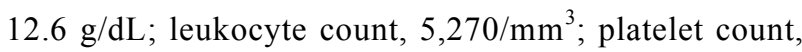
$50,000 / \mathrm{mm}^{3}$; aspartate aminotransferase (AST), $34 \mathrm{IU} / \mathrm{L}$; alanine aminotransferase (ALT), $21 \mathrm{IU} / \mathrm{L}$; total bilirubin, 5.7 $\mathrm{mg} / \mathrm{dL}$; prothrombin time, $20.8 \mathrm{~s}$; alkaline phosphatase, 404 IU/L; and ammonia, $45 \mu \mathrm{M} / \mathrm{L}$. Rib series and chest computed tomography (CT) scans showed multiple rib fractures and hemothorax. Therefore, we performed thoracostomy. Dysarthria and ataxia occurred on day 25 after thoracostomy (total dose of metronidazole, $30 \mathrm{~g}$ ), and a state of confusion was reported on day 26. Subsequently, we performed an arterial blood test and an electrolyte test and measured the blood ammonia levels. The results of these examinations were within normal limits. Brain CT scans showed increased density in the corpus callosum. Brain MRI, T2-weighted and FLAIR images showed a high signal intensity in the dentate
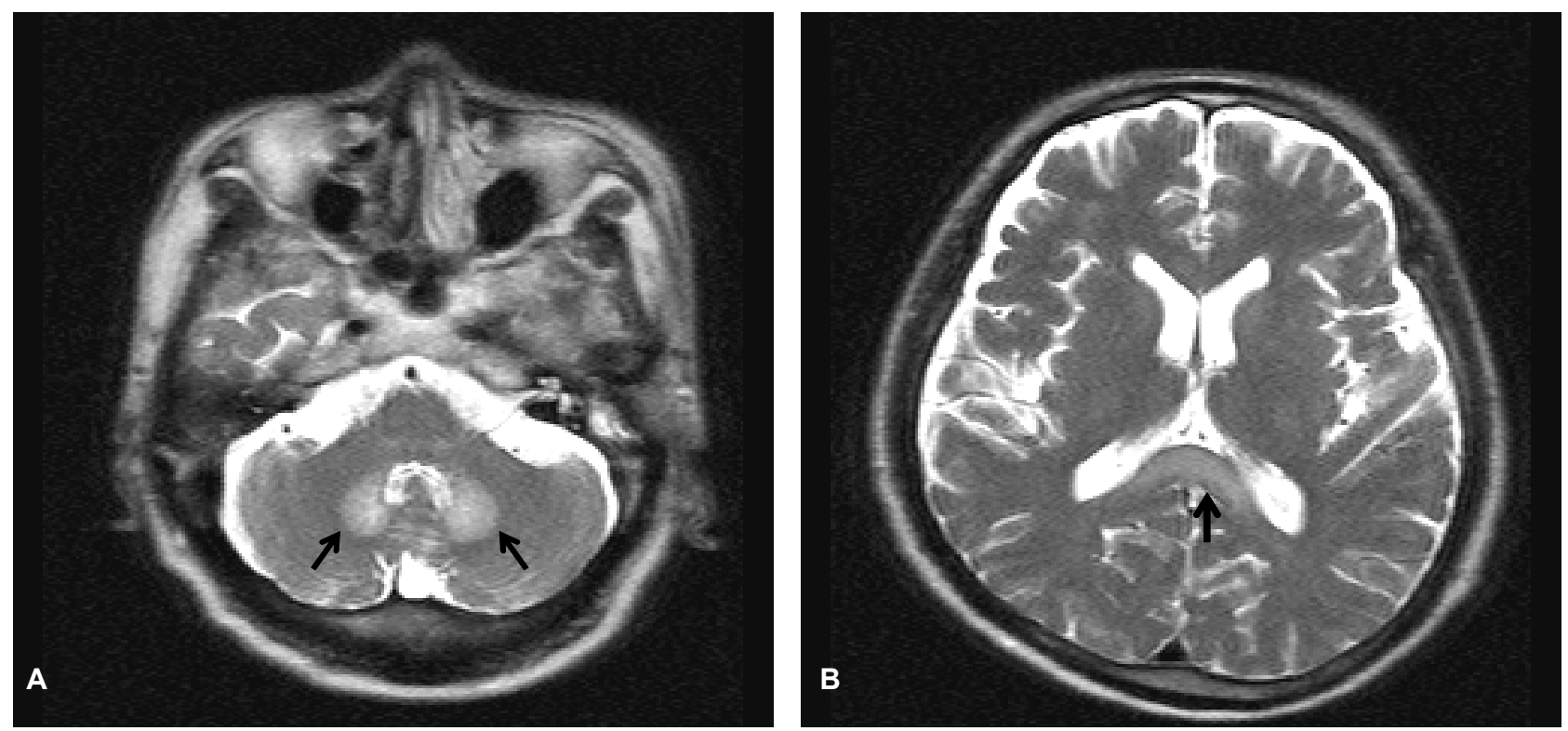

Figure 1. T2-weighted brain magnetic resonance images showed symmetrically increased signal intensity (arrows) in both the dentate nucleus (A) and the splenium of the corpus callosum (B).
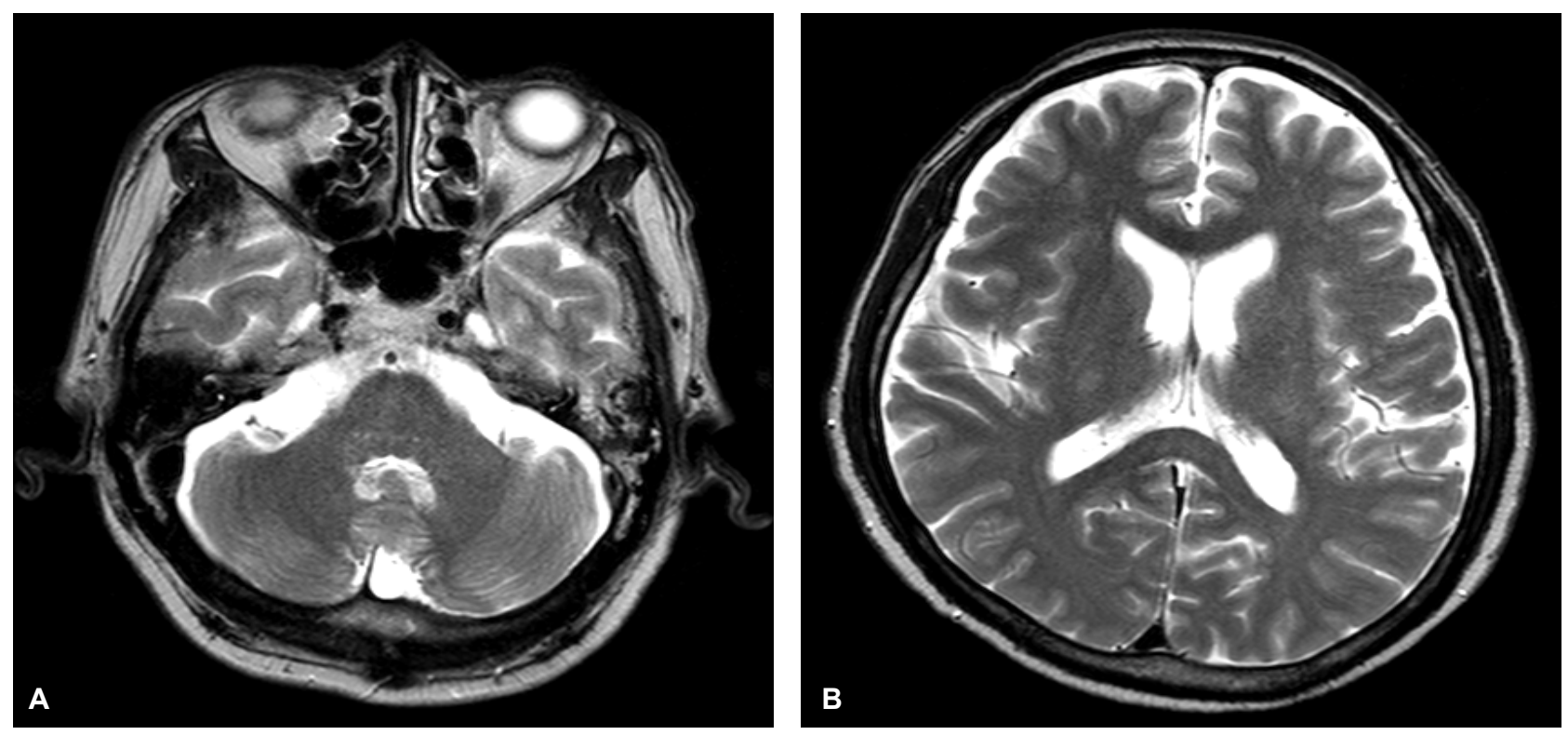

Figure 2. Five weeks after discontinuation of metronidazole, T2-weighted brain magnetic resonance images showed complete resolution of the high signal intensity in both the dentate nucleus (A) and the splenium of the corpus callosum (B). 
nuclei on both sides and in the splenium of the corpus callosum, and the diffusion-weighted images showed vasogenic edematous lesions in a part of the corpus callosum. Therefore, metronidazoleinduced encephalopathy was suspected (Fig. 1). In addition, the T1-weighted image showed high signal intensity in the superior ganglion on both sides and in the cerebral peduncle, suggesting hepatic encephalopathy. The patient was transferred to the liver disease clinic, metronidazole was discontinued, and conservative treatment for liver cirrhosis and encephalopathy was initiated. One day after discontinuation of metronidazole, he showed improvement in dysarthria and the state of consciousness, and after 2 days, he showed a normal state of consciousness. Two weeks after stopping metronidazole administration, weakness of the lower extremities reduced. In follow-up brain MRI, which was performed after 5 weeks, we confirmed the disappearance of high signal intensity in the superior ganglion on both sides and in the splenium of the corpus callosum in T2-weighted and FLAIR images (Fig. 2). After 1 month, the patient was able to walk, and he is currently under outpatient follow-up. He is also receiving lactulose and rifaximin to control the repeated episodes of hepatic encephalopathy.

\section{DISCUSSION}

Metronidazole, a drug used for the treatment of various abscesses caused by infection with anaerobic bacteria, can decrease ammonia production by inhibiting the growth of intestinal bacteria. Therefore, it is also used to treat hepatic encephalopathy that cannot be controlled by lactulose. The common adverse events associated with metronidazole treatment are nausea, bad taste, headache, and dizziness, and in rare cases, encephalopathy. The occurrence of encephalopathy depends on the cumulative dose, and encephalopathy presents with sudden symptoms such as cerebellar dysfunction, ataxia, dysarthria, convulsion, encephalopathy, and vertigo. ${ }^{3}$ In the Korean medical database, 11 cases of metronidazoleinduced neuropathy have been reported. In these cases, the symptoms appeared after $21 \mathrm{~g}$ to $135 \mathrm{~g}$ of metronidazole had been administered. ${ }^{4-10}$ Three of these 11 patients showed peripheral neuropathy, ${ }^{4}$ while the other 9 showed encephalopathy. $^{5-10}$

With regard to its pharmacological action, metronidazole shows microbicidal activity against anaerobic bacteria by blocking the action of a DNA repair enzyme. ${ }^{3}$ Approximately,
$30-60 \%$ of metronidazole is metabolized in the liver, and the metabolite has antimicrobial activity, passes through the cerebrospinal fluid, and crosses the blood-brain barrier. ${ }^{1}$ The half-life of the drug is 6-8 hours, but in hepatic encephalopathy patients, its clearance rate is reduced by one-third because of diminished liver function; as a result, the half-life of the drug is increased approximately 3 -folds in these patients. ${ }^{11}$ Accordingly, in patients with liver cirrhosis, the drug accumulates in the blood faster, which raises blood levels and causes encephalopathy symptoms such as decreased consciousness. ${ }^{9}$ Even our patient had liver cirrhosis, he experienced cerebellar dysfunction symptoms such as ataxia and encephalopathy symptoms such as dysarthria and confusion after the administration of a relatively low cumulative dose of $30 \mathrm{~g}$. Another study has reported that encephalopathy occurred earlier in patients with renal dysfunction $(21 \mathrm{~g}$ administered over a 2 -week period). ${ }^{7}$ Although previous studies have shown that the half-life of metronidazole does not increase when hemodialysis is performed, ${ }^{12}$ the half-life of hydroxyl metabolites is believed to increase, and their accumulation leads to encephalopathy at a relatively low cumulative dose. ${ }^{13}$ Accordingly, it is necessary to limit the total cumulative dose to less than $20 \mathrm{~g}$. Furthermore, when metronidazole is used, the patient's neurological symptoms should be monitored closely.

Metronidazole-induced encephalopathy may cause symptoms such as dysarthria, gait disturbance, dizziness, and decreased consciousness, and can be accompanied by a tingling sensation in the distal extremities. Our patient also showed the typical symptoms such as ataxia, dysarthria, and confusion, but he did not experience paresthesia in the distal extremities. In most cases of metronidazole-induced encephalopathy, including our case, these symptoms disappear completely within a few days after discontinuation of therapy. However, a recent study reported a case in which the patient died of encephalopathy. ${ }^{11}$ The patient took metronidazole for 10 weeks, and the total cumulative dose was $132 \mathrm{~g}$. The drug administration was stopped after symptoms of encephalopathy appeared, however, the patient's symptoms did not improve and died 8 weeks after the onset of the symptoms. As evidenced by this case, metronidazoleinduced encephalopathy is not always reversible and may result in a fatal outcome. Therefore, when metronidazole is administered, the dose and duration of treatment should be limited. 
Metronidazole-induced encephalopathy can be definitively diagnosed if the patient experiences new neurological disorders or if brain MRI shows characteristic lesions in a patient who is receiving metronidazole. ${ }^{14}$ Patients with metronidazole-induced encephalopathy typically show characteristic symmetric lesions in brain MRI, and such lesions are usually limited to the region around the cerebellar dentate nuclei. However, some rare cases may also show multiple symmetric lesions in the corpus callosum, midbrain, pons, white matter, basal ganglia, etc., and high signal intensity in T2-weighted, FLAIR, and diffusion-weighted images. Since the brain MRI findings of our patient also showed a high signal intensity (T2-weighted image) in both the dentate nuclei and the corpus callosum, we suspected the condition to be metronidazole-induced encephalopathy. The high signal intensity seen on T2-weighted brain MRI images is believed to appear because the 5-nitroimidazole derivative generates toxic radicals via the oxidation of neurotransmitters such as catecholamines, and the radicals damage metallic ion-containing nerve cells in the brain, thereby increasing water content and causing axonal swelling. ${ }^{15}$ Low signal intensity was rarely observed in apparent diffusion coefficient maps. In one such case, cytotoxic edema was caused by a mechanism other than cell ischemia and had a poor prognosis. ${ }^{16}$ When MRI shows multiple symmetric lesions in the white matter and the gray matter without mass effect, it is necessary to differentiate this condition from hypoglycemia, electrolyte disturbance, trauma, acute disseminated encephalomyelitis, inflammatory disease, diseases that affect the nervous system or metabolism, as well as from drug toxicity caused by drugs such as anticonvulsants. ${ }^{15}$ Among the 10 cases of metronidazole-induced encephalopathy reported in Korea, 8 (including our case) showed characteristic MRI findings (high signal intensity in cerebellar dentate nucleus, corpus callosum, midbrain, and basal ganglia on T2- weighted images); however, the other 2 patients did not show such findings, and they were diagnosed on the basis of clinical outcomes. In all cases that underwent follow-up MRI, the characteristic high signal intensity had disappeared.

Metronidazole-induced encephalopathy is treated with discontinuation of drug therapy. After discontinuation of drug therapy, most symptoms improve, but occasionally, the symptoms of peripheral neuropathy persist in the distal extremities. ${ }^{4}$

In conclusion, metronidazole is an effective drug for the treatment of various abscesses caused by infection with anaerobic bacteria and hepatic encephalopathy not controlled by administration of lactulose. However, for older patients, patients with liver cirrhosis, and patients with renal failure who have reduced drug metabolism, it is necessary to restrict the total dose and the duration of administration and consider the cumulative dose. Caution should be exercised, particularly for cases of liver cirrhosis that are similar to our case, because hepatic encephalopathy may occur even after lowdose metronidazole administration.

\section{REFERENCES}

1. Lamp KC, Freeman CD, Klutman NE, Lacy MK. Pharmacokinetics and pharmacodynamics of the nitroimidazole antimicrobials. Clin Pharmacokinet 1999;36:353-373.

2. Uhl MD, Riely CA. Metronidazole in treating portosystemic encephalopathy. Ann Intern Med 1996;124:455.

3. Kusumi RK, Plouffe JF, Wyatt RH, Fass RJ. Central nervous system toxicity associated with metronidazole therapy. Ann Intern Med 1980;93:59-60.

4. Sunwoo IN, Kim SM, Choi MS, Chang J, Chung JB, Chon CY. Metronidazole neuropathy: report of three cases. J Korean Med Assoc 1988;31:561-566.

5. Ji KH, Lee J, Yun $\mathrm{CH}$, Ha CK. Reversible metronidazole-induced encephalopathy. J Korean Soc Clin Toxicol 2006;4:131-136.

6. Huh SY, Kim JK, Kim MJ, Yoo BG, Kim KS, Lee JH . Reversible encephalopathy induced by metronidazole. J Korean Geriatr Soc 2008; $12: 176-178$.

7. Lee JH, Kim MO, Choi SY, Choi HJ, Kim HJ, Kang KW, et al. Metronidazole-induced toxic encephalopathy in a hemodialysis patient: a case report. Korean J Nephrol 2008;27:743-746 .

8. Kwon KY, Lee DK, Lee KH, Cho KH, Lee E, Chung SJ . Two cases of metronidazole-induced neurotoxicity lacking of clinico-radiological correlation. J Korean Neurol Assoc 2006;24:581-584.

9. Kim KH, Choi JW, Lee JY, Kim TD, Paek JH, Lee EJ, et al. Two cases of metronidazole-induced encephalopathy. Korean J Gastroenterol 2005; 45:195-200

10. Kim DS, Jung JW, Kim JY, Kim JH, Kim EK, Kim SE . Reversible MRI findings in metronidazole-induced cerebellar dysfunction. J Korean Neurol Assoc 1999;17:904-907.

11. Groothoff MV, Hofmeijer J, Sikma MA, Meulenbelt J. Irreversible encephalopathy after treatment with high-dose intravenous metronidazole. Clin Ther 2010;32:60-64.

12. Somogyi A, Kong C, Sabto J, Gurr FW, Spicer WJ, McLean AJ. Disposition and removal of metronidazole in patients undergoing haemodialysis. Eur J Clin Pharmacol 1983;25:683-687.

13. Lau AH, Chang CW, Sabatini S. Hemodialysis clearance of metronidazole and its metabolites. Antimicrob Agents Chemother 1986;29:235-238.

14. Bradley WG, Karlsson IJ, Rassol CG. Metronidazole neuropathy. Br Med J 1977;2:610-611.

15. Ahmed A, Loes DJ, Bressler EL. Reversible magnetic resonance imaging findings in metronidazole-induced encephalopathy. Neurology 1995;45:588-589.

16. Kim DW, Park JM, Yoon BW, Baek MJ, Kim JE, Kim S. Metronidazole-induced encephalopathy. J Neurol Sci 2004;224: 107-111. 